\title{
Editorials
}

\section{Medication safety in anesthetic practice: first do no harm}

A

NESTHESIOLOGISTS are proud of the remarkable improvements in patient safety that have occurred in the past $20 \mathrm{yr}$. Mortality rates have decreased from the order of one per 5,000 to perhaps as few as one per $200,000-300,000$ anesthetics administered. ${ }^{1}$ However, to achieve additional incremental gains, the remaining obstacles to improving patient safety must be identified and corrected. If patient safety is defined as freedom from accidental injury, then several studies suggest that medication error has become a leading cause of adverse events during anesthesia. For example, an analysis of critical incidents by Cooper et al., (1978 and 1984) demonstrated that the total number of medication-related events (including syringe swaps, drug ampoule swaps, overdose, or wrong choice of drug) far exceeded the next most frequent problem, disconnection of the breathing circuit. ${ }^{2,3}$ Similarly, an analysis of the first 2,000 events reported to the Australia Incident Monitoring Study (AIMS) revealed that the "wrong drug" was a common problem. ${ }^{4}$

Multiple factors contribute to the increased risk of medication error for patients undergoing anesthesia. These patients routinely receive potent drugs that carry the risk of serious injury or death when administered in excessive doses or without adequate patient support systems. The drugs are delivered in a dynamic, complex environment in which the anesthesiologist's attention is often distracted by other patient concerns. Furthermore, a single individual performs the tasks of prescribing, dispensing, administering and monitoring the drugs. Thus, the safeguards that are present on the wards (such as the review of medication orders by nurses or pharmacists) are lacking. Also, the number of errors increases with the number of drugs administered and patients frequently receive many drugs over a short period of time. Most anesthesiolo- gists report being involved in at least one medication error. ${ }^{5}$ Given so many opportunities for error and a system that provides few safeguards, it can be expected that medication errors in anesthesia will occur frequently. Perhaps it is more remarkable that there are not more catastrophic outcomes.

The impact of preventable adverse events was recently highlighted by the landmark report entitled "To Err is Human: Building a Safer Health System" from the National Academy of Science's Institute of Medicine. ${ }^{6}$ This report estimated that between 44,000 to 98,000 Americans die each year as the result of a medical error. Medication errors figured prominently in these adverse events. The report also acknowledged the lack of prospective data regarding the factors that contribute to medical error or successful strategies for risk reduction. This paucity of data is the reason why an article published in this issue of the Journal is important and noteworthy. Drs. Fasting and Gisvold are to be congratulated for providing new knowledge by estimating the incidence of drug error during anesthesia. ${ }^{7}$ However, the analysis and conclusions of their important findings require careful reconsideration.

The objective of the study was to determine the impact of two interventions on the incidence of medication error during anesthesia: 1) the replacement of text labels with colour-coded labels for syringes and 2) educational sessions that raised awareness about medication error during anesthesia. The number of errors that occurred during the 18 -month period, before and after the interventions, was determined by a selfreporting method. The major finding of the study was that the rate of drug error decreased from 40 per 28,971 cases ( 1.38 per 1000 cases) to 23 in 26,455 cases $(0.869$ per 1000 cases), following the interventions (a 37\% reduction in error rate was observed). These data confirm that the incidence of drug error

Address correspondence to: Department of Anesthesia, Sunnybrook and Women's College Health Science Centre, University of Toronto, 2075 Bayview Avenue, Toronto, Ontario, M4N 3M5 Canada. Phone: 416-978-0574; Fax: 416-978-4940; E-mail: beverley.orser@utoronto.ca 
during anesthesia is low and most errors reportedly did not result in harm. However, some errors were associated with significant morbidity. For example, 12 patients received muscle relaxants while awake.

Based on a Chi-squared analysis, the authors concluded that the pre- and post-intervention rates of medication error were not statistically different. However, the $37 \%$ reduction in the rate of error that occurred followed the interventions requires further analysis. The purpose of the study was "To evaluate whether a change to color coded syringe labels, along with education, could reduce the problem of drug errors". Therefore, it would have been reasonable to test a one-sided hypothesis ergo to have assumed that the interventions were unlikely to cause an increase in the incidence of medication error. A one-tailed statistical test, as previously suggested by Kleinbaum et al., (1982), can be applied. ${ }^{8}$ Using a one-sided Z-test, the pre-intervention rate is discernibly greater than after the interventions $(P=0.037)$. Thus, these data provide the first evidence that education and/or colourcoded syringe labels decrease the overall rate of drug error during anesthesia. The study also showed that the interventions were associated with a decrease in the incidence of ampoule swaps.

The results of this study are exciting news and indicate that the two simple interventions can reduce the risk of accidental injury during anesthesia (education and/or colour-coded syringe labels). Furthermore, it is advisable that they be implemented. Until future studies identify other strategies for risk reduction, the following 17 recommendations are intended to improve the safety of medication delivery systems for patients undergoing anesthesia.

1. Recognize medication error as a leading cause of adverse events in anesthetic practice.

2. Educate and increase awareness about medication safety.

3. Create an environment that encourages the identification, discussion and correction of all errors. Urge that ALL medication errors and NEAR medication errors be reported via the Quality Assurance processes.

4. Practice and teach to Read/Read/Read/Read the labels on drug ampoules and vials (when taking the drug from the anesthetic cart, before and after drawing it into a syringe and prior to administration).

5. Utilize self-adhesive syringe labels that conform to the standards established by the CSA International (Canadian Standards Association) Z264.3-98.

6. Pre-label syringes before drawing up the drug.
7. Group purchasers of medications should demand that the labels on ampoules, vials and pre-filled syringes comply with CSA International standard CAN/CSA Z264.2-99.

8. Drug bags should be labeled using large fluorescent labels that indicate the concentration, total dose per total volume, name of the individual that prepared the solution, and date of preparation.

9. Designate a Medication Safety Officer.

10. Utilize strategies that reduce memory requirements for pharmacological information and make that information available at the point of patient care. For example, ePocrates $q R x$ (www.epocrates.com) is a drug information guide designed for Palm Computing Platforms.

11. Limit the number of medications in anesthetic drug carts.

12. Review carefully the set of prepared drugs during any exchange of anesthesia personnel.

13. Inform members of the department of anesthesiology when a new drug product is introduced into the anesthetic drug cart, operating rooms or critical care areas.

14. Avoid look-alike ampoule and vials in the anesthetic drug carts.

15. Anesthetic drug carts used within a single institution should have a similar design.

16. Keep the work surface of the anesthetic drug cart tidy and organized.

17. Report medication errors to the Institute of Safe Medication Practice-Canada (www.ISMPCanada.com).

By implementing such strategies, hopefully a leading cause of adverse anesthetic events can be corrected. Further studies designed to demonstrate whether these recommendations improve patient outcome are to be encouraged.

\section{Acknowledgements}

The author expresses appreciation to Dr. John P. Szalai for assistance with the statistical analysis and Drs. Richard Hall, Jeffrey B. Cooper, Ellison C. Pierce and Daisy Joo for reviewing the manuscript. A Career Scientist Award from the Ontario Ministry of Health and Canadian Institutes of Health Research supports Dr. Orser's research. 


\section{La sécurité d'emploi des médicaments anesthésiques: d'abord ne pas nuire}

Les anesthésiologistes sont fiers de l'évolution remarquable apportée à la sécurité du patient pendant les 20 dernières années. En effet, les taux de mortalité ont baissé de un sur 5000 à environ aussi peu que un sur 200000 - 300000 cas d'anesthésie. ${ }^{1}$ Cependant, pour faire de nouveaux gains, on doit identifier et corriger les obstacles résiduels. Partant de la définition de la sécurité comme l'absence de lésion accidentelle, certaines études suggèrent que l'erreur de médication constitue la cause principale des incidents qui surviennent pendant l'anesthésie. Par exemple, une analyse des incidents majeurs de Cooper et coll., (1978 et 1984) a démontré que le nombre total d'incidents reliés à la médication (y compris l'échange de seringue, d'ampoule, la surdose ou le mauvais choix de médicament) excède de loin le deuxième problème qui se présente fréquemment, l'interruption du circuit respiratoire. $^{2,3}$ De même, une analyse des 2000 premiers incidents rapportés par l'Australia Incident Monitoring Study (AIMS) a révélé que le "mauvais médicament" a représenté le problème le plus fréquent. ${ }^{4}$

De multiple facteurs contribuent à l'augmentation des risques d'erreurs de médication chez les patients qui subissent une anesthésie. Ces patients reçoivent des médicaments puissants qui comportent des risques mortels ou de lésions sérieuses s'ils sont administrés en doses excessives ou sans soutien adéquat du patient. Les médicaments sont administrés dans un environnement dynamique et complexe où l'attention de l'anesthésiologiste est souvent détournée par d'autres préoccupations reliées au patient. De plus, c'est la même personne qui prescrit, distribue, administre les médicaments et en surveille l'action. Alors, les mesures de sécurité présentes à l'étage (comme la vérification de l'ordonnance par les infirmières ou le pharmacien) sont absentes. Aussi, le nombre d'erreurs augmente avec le nombre de médicaments administrés et avec le fait que des patients reçoivent de nombreux médicaments pendant une courte période de temps. La plupart des anesthésiologistes ont rapporté avoir été impliqués au moins une fois dans une erreur de médication. ${ }^{5}$ Étant donné les nombreuses occasions d'erreurs possibles et le peu de mesures de contrôle du système, on peut s'attendre que des erreurs de médi- cation anesthésique surviennent fréquemment. Peut-être est-il plus remarquable encore qu'il y ait aussi peu d'incidents catastrophiques.

L'impact des incidents évitables a été récemment mis en lumière par le rapport clé intitulé "To Err is Human: Building a Safer Health System" de la National Academy of Sciences's Institutes of Medicine. ${ }^{6}$ Ce rapport a estimé qu'entre 44000 à 98000 Américains meurent chaque année des suites d'erreurs médicales. Les erreurs de médication figurent en tête de liste de ces incidents. Le rapport reconnait aussi un manque de données prospectives sur les facteurs qui contribuent à l'erreur médicale ou sur le succès des stratégies de réduction des risques. Étant donné la pauvreté des données, la publication d'un article dans le présent numéro du Journal constitue un fait marquant. Il faut donc reconnaître la contribution des docteurs Fasting et Gisvold qui ont cherché à fournir une estimation de l'incidence des erreurs de médication pendant l'anesthésie? Toutefois, l'analyse et les conclusions de leurs importantes données demandent une reconsidération attentive.

L'objectif de l'étude était de déterminer l'effet de deux interventions sur l'incidence d'erreur de médication pendant l'anesthésie:1) le remplacement des étiquettes avec texte par des étiquettes avec un code de couleurs et 2) des sessions de formation pour sensibiliser sur les erreurs de médication pendant l'anesthésie. Le nombre d'erreurs survenues pendant les 18 mois qui ont précédé et suivi les interventions a été établi par une méthode d'auto-divulgation des erreurs. La principale constatation de l'étude a été que le taux d'erreur de médicament a chuté, passant de 40 pour 28971 cas $(1,38$ pour 1000 cas) à 23 pour 26 455 cas $(0,869$ pour 1000 cas $)$, à la suite des interventions (on a observé une réduction des erreurs de $37 \%$ ). Ces données confirment que l'incidence d'erreur de médicament pendant l'anesthésie est basse et que la plupart des erreurs rapportées ne causent pas de préjudice. Cependant, certaines erreurs ont été associées à une morbidité significative. Par exemple, 12 patients ont reçu un myorelaxant alors qu'ils étaient éveillés.

Se fondant sur une analyse du $\mathrm{Khi}^{2}$, les auteurs ont conclu que les taux d'erreurs de médicaments avant et après les interventions n'ont pas présenté de différence statistique significative. Cependant, la réduction de $37 \%$ du taux d'erreur survenue à la suite des interventions exige une analyse supplémentaire. Le but de l'étude était d'“évaluer si l'adoption d'étiquettes marquées d'un code de couleurs, accompagnée d'une formation, pouvait réduire le problème des erreurs de médicaments." Il aurait été souhaitable de tester une hypothèse unilatérale, donc de présumer qu'il était peu probable que les interventions causent d'augmentation de l'incidence d'erreur de médicament. Un test statistique unilatéral, comme l'ont déjà suggéré Kleinbaum et coll., (1982), peut s'appliquer. ${ }^{8}$ 
En utilisant un test $\mathrm{Z}$ unilatéral, le taux pré-intervention est sensiblement plus élevé que le taux postintervention $(P$ $=0,037)$. Ainsi, ces données fournissent la première évidence qu'une formation et/ou des étiquettes de seringue codées en couleurs font baisser le taux global d'erreur de médicament pendant l'anesthésie. L'étude a aussi montré que les interventions ont été associées à une baisse de l'incidence d'échange d'ampoules.

Les résultats de cette étude sont encourageants et indiquent que deux interventions toutes simples peuvent réduire le risque de lésion accidentelle pendant l'anesthésie. Il serait donc sage de les appliquer. Jusqu'à ce que d'autres études déterminent d'autres stratégies de réduction des risques, les 17 recommandations suivantes visent à concevoir des systèmes d'administration de médicaments plus sûrs pour les patients qui subissent une anesthésie.

1. Reconnaitre l'erreur de médicament comme la cause principale d'incidents en anesthésie.

2. Eduquer les cliniciens et les sensibiliser à la sécurité de la médication.

3. Créer un environnement qui encourage d'identifier les erreurs, d'en discuter et de les corriger toutes. Insister pour que TOUTES et les QUASI-erreurs de médication soient rapportées par une démarche d'Assurance qualité.

4. Pratiquer et enseigner à Lire/Lire/Lire/Lire les étiquettes sur les ampoules et les flacons de médicaments (quand on prend le médicament sur le chariot d'anesthésie, avant et après l'avoir aspiré dans la seringue et avant de l'administrer).

5. Utiliser des étiquettes de seringues auto-collantes qui répondent aux normes établies par l'Association canadienne de normalisation (ACNOR) Z264.3-98.

6. Pré-étiqueter les seringues avant de avant de les remplir.

7. Exiger que les achats de groupe comportent des étiquettes d'ampoules, de flacons et de seringues pré-remplies conformes aux normes de l'ACNOR CAN/CSA Z264.2-99.

8. Proposer que les sacs de médicaments aient de grandes étiquettes fluorescentes qui indiquent la concentration, la dose totale par volume total, le nom de la personne qui a préparé la solution et la date de la préparation.

9. Désigner un responsable de la sécurité la sécurité des médications.

10. Utiliser des stratégies qui réduisent la nécessité de mémoriser les informations pharmacologiques et présenter ces renseignements sur les lieux où sont rendus les soins. Par exemple, ePocrates $q R x$ (www.epocrates.com) est un guide d'information sur les médicaments conçu pour Palm Computing Platforms (ordi- nateurs de poche).

11. Limiter le nombre de médicaments sur les chariots d'anesthésie.

12. Revoir attentivement l'ensemble des médicaments préparés pendant tout changement de personnel d'anesthésie.

13. Informer les membres du service d'anesthésiologie lorsqu'un nouveau médicament est ajouté au chariot d'anesthésie, dans les salles d'opération ou aux soins intensifs.

14. Éviter les ampoules et les flacons semblables sur les chariots d'anesthésie.

15. Adopter un modèle similaire pour tous les chariots de médicaments anesthésiques à l'intérieur d'une même institution.

16. Garder la surface de travail du chariot propre et bien rangée.

17. Rapporter les erreurs de médication à l'Institute of Safe Medication Practice-Canada (www.ISMP-Canada.com).

L'implantation de ces stratégies permettra, nous l'espérons, de corriger la principale cause des incidents anesthésiques. D'autres études, conçues pour faire la preuve de l'efficacité de ces recommandations sur l'évolution du patient, doivent donc être favorisées.

\section{References}

1 Sentinel events: Approaches to error reduction and prevention. Jt Comm J Qual Improv 1998; 24: 175-86.

2 Cooper JB, Newbower RS, Long CD, McPeek B. Preventable anesthesia mishaps: a study of human factors. Anesthesiology 1978; 49: 399-406.

3 Cooper JB, Newbower RS, Kitz RJ. An analysis of major errors and equipment failures in anesthesia management: considerations for prevention and detection. Anesthesiology 1984; 60: 34-42.

4 Currie M, Mackay P, Morgan C, et al. The Australian Incident Monitoring Study. The "wrong drug" problem in anaesthesia: an analysis of 2000 incident reports. Anaesth Intensive Care 1993; 21: 596-601.

5 Orser BA, Chen RJB, $\Upsilon_{e e} D A$ Medication errors in anesthetic practice: a survey of 687 practitioners. Can J Anesth (in press).

6 Kohn LT, Corrigan JM, Donaldson MS. To Err is Human: Building a Safer Health System. Washington DC: National Academy Press, 1999.

7 Fasting S, Gisvold SE. Adverse drug errors in anesthesia, and the impact of coloured syringe labels Can J Anesth 2000; 47: 1060-67.

8 Kleinbaum David G, Kooper Lawrence L, Morgenstern Hall. Epidemiological Research: Principals and Quantitative Methods. New York NY; Van Nostrand Reinhold Company, 1982. 